\title{
Correction to: Ledipasvir-Sofosbuvir for 8 Weeks in Non-Cirrhotic Patients with Previously Untreated Genotype 1 HCV Infection \pm HIV-1 Co-Infection
}

\author{
Vasily Isakov ${ }^{1}$ - Natalia Gankina ${ }^{2} \cdot$ Viacheslav Morozov $^{3} \cdot$ Kathryn Kersey $^{4} \cdot$ Sophia Lu $^{4} \cdot$ Anu Osinusi $^{4}$.

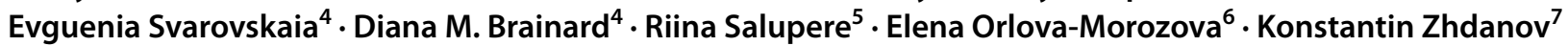

Published online: 23 February 2021

(c) Springer Nature Switzerland AG 2021

\section{Correction to: Clin Drug Investig (2018) 38:239-247 https://doi.org/10.1007/s40261-017-0606-0}

The original version of this article unfortunately contained a mistake. The correct information is given below.

Page 242, Section 3.3, 1st paragraph, which previously read:

Baseline virologic resistance was assessed in all patients, except for one patient with HCV mono-infection who had other virologic outcome $(n=124)$. NS5A was successfully deep-sequenced in all 124 patients. At baseline, 2\% (3/124) of patients had NS5A RASs using a $15 \%$ assay cut-off; one genotype $1 \mathrm{~b}$-infected patient had the L31M RAS, and two

The original article can be found online at https://doi.org/10.1007/ s40261-017-0606-0.

Vasily Isakov

vasily.isakov@gmail.com

1 Department of Gastroenterology and Hepatology, Federal Research Center for Nutrition, Biotechnology and Food Safety, Kashirskoe shosse, 21, Moscow 115446, Russian Federation

2 Clinical and Diagnostic Department, Krasnoyarsk Regional Center for Prevention and Control of AIDS and Infectious Diseases, Krasnoyarsk, Russian Federation

3 Hepatolog, LLC, Samara, Russian Federation

4 Clinical Research, Gilead Sciences, Foster City, CA, USA

5 Department of Internal Medicine, Tartu University Hospital, Tartu, Estonia

6 Moscow Regional Center for Prevention and Control of AIDS and Infectious Diseases, Moscow, Russian Federation

7 Department of Infectious Diseases, Military Medical Academy, St Petersburg, Russian Federation genotype 1b-infected patients had the Y93H RAS. NS5B was successfully deep-sequenced in 123 patients; NS5B amplification failed in one patient co-infected with genotype $1 \mathrm{HCV}$ and HIV. Thirty-four genotype $1 \mathrm{~b}$-infected patients had L159F NS5B NI RAS present at baseline using a 15\% assay cut-off. All patients with baseline RASs achieved SVR12. None of the patients who relapsed had any RASs at baseline or time of virologic failure.

\section{Should read:}

Baseline virologic resistance was assessed in all patients, except for one patient with HCV mono-infection who had other virologic outcome ( $n=125)$. NS5A was successfully deep-sequenced in all 125 patients. At baseline, 3\% (4/125) of patients had NS5A RASs using a 15\% assay cut-off; one genotype $1 \mathrm{~b}$-infected patient had the L31M RAS, and three genotype 1b-infected patients had the Y93H RAS. NS5B was successfully deep-sequenced in 124 patients; NS5B amplification failed in one patient co-infected with genotype $1 \mathrm{HCV}$ and HIV. Thirty-five genotype $1 \mathrm{~b}$-infected patients had L159F NS5B NI RAS present at baseline using a 15\% assay cut-off. All patients with baseline RASs achieved SVR12. None of the patients who relapsed had any RASs at baseline or time of virologic failure. 